\title{
Calorie restriction prevents the development of insulin resistance and impaired lipid metabolism in gestational diabetes offspring
}

\author{
Tao Li', Keyang Chen², Gang Liu', Li-Ping Huang' ${ }^{1}$, Long Chen', Qiu-Wei Wang', Chuan-Lai Hu' and Li-Juan Hou'
}

BACKGROUND: Gestational diabetes mellitus (GDM) has long-lasting influence on offspring, which is associated with increased risks of insulin resistance, obesity, and type II diabetes mellitus. Calorie restriction (CR) is one of the most common and available nutritional interventions to prevent obesity and diabetes. We are trying to explore the effect of CR on GDM offspring.

METHODS: The streptozotocin was used to stimulate C57BL/6J mice to develop GDM, a number of metabolic characteristics and related protein expressions were determined in GDM offspring that were fed ad-libitum or treated with calorie restriction.

RESULTS: CR reduced body weight and glucose levels in GDM offspring. CR modulated the lipid metabolism by decreasing triglyceride and cholesterol levels in plasma. We also found that the effect of CR on insulin sensitivity may involve in signaling pathway through the regulations of phosphatase and tensin homologue deleted on chromosome 10 (PTEN) and protein kinase B (Akt).

CONCLUSION: GDM is a high risk factor for GDM offspring to develop insulin resistance, while CR could ameliorate this adverse outcome. Moreover, the specific decrease in PTEN activation and increase in Akt phosphorylation in livers of GDM offspring with CR improved insulin sensitivity and lipid metabolism.

\footnotetext{
G estational diabetes mellitus (GDM) is defined as the onset of any degree of glucose intolerance during pregnancy, and is associated with increased fetal-maternal morbidity as well as long-term complications in mothers and offspring (1). Besides, women with GDM have a large increased risk of prenatal morbidity and a considerably elevated risk of impaired glucose tolerance and type II diabetes in the years following pregnancy (2). Moreover, children of women with GDM are more likely to be obese, impaired glucose tolerance and type II diabetes in the following years (3-5). Impaired glucose tolerance and obesity are two to three times more frequent in children from mothers who had diabetes during pregnancy than
}

in children from mothers who developed diabetes after pregnancy (6).

Diet problem is one of the main risk factors for gestational diabetes. A calorie-reduced diet is a primary therapy for people with insulin resistance and obesity, even moderate reduction of calorie during a 3 to 6 -d hospitalization decreases insulin levels, postprandial blood glucose (7) and improves hepatic and skeletal muscle insulin sensitivity (8). This treatment is called calorie restriction (CR). CR can be broadly defined as restricting the diet of an organism to fewer calories (20-50\%) rather than ad-libitum-feeding without altering the levels of amino acids, vitamins, and minerals (9-11).

Although calorie restriction is the most economical and convenient intervention to extend lifespan and delay the negative physiologic consequences of chronic diseases associated with aging, the relationship between $\mathrm{CR}$ and insulin signaling and lipid metabolism remain uncertain. Recently, large experimental and clinical studies show that insulin sensitivity and a variety of cardiovascular diseases are significantly ameliorated by CR $(12,13)$, in which the underlying molecular mechanism responsible for the increased insulin sensitivity may be associated with increased activation of certain elements in insulin signaling $(11,14,15)$. However we did not know whether or not $\mathrm{CR}$ can reduce the risk of adverse outcomes in GDM offspring. In addition, we did not know how insulin signaling pathway in GDM offspring would be imposed by CR.

The purpose of this study is to evaluate whether caloric restriction can reverse the adverse outcome in GDM offspring. To address this question, a number of metabolic characteristics, including blood glucose, insulin, cholesterol (TCH), triglyceride (TG), high-density lipoprotein (HDL)cholesterol (HDL-C), and low-density lipoprotein (LDL)cholesterol (LDL-C) were determined in GDM offspring that received ad libitum feeding or calorie restriction. In addition, protein expression and functionality of the PI3-kinase/Akt pathways including phosphotidylinositol-3-kinase (PI3K), Akt, phosphatase and tensin homologue deleted on chromosome 10 (PTEN), Protein tyrosine phosphatase 1B (PTP1B) and lipid metabolism related protein ATP citrate lyase (ACL)

\footnotetext{
The first two authors contributed equally to this work.

'Department of Nutrition and Food Safety, Anhui Medical University School of Public Health, Anhui, China; ${ }^{2}$ Department of Public Health Inspection and Quarantine Science, Anhui Medical University, Anhui, China. Correspondence: Chuan-Lai Hu (huchuanlai@ahmu.edu.cn)

Received 21 May 2016; accepted 4 December 2016; advance online publication 1 March 2017. doi:10.1038/pr.2016.273
} 
were assayed. Furthermore the phosphoprotein including p-PI3-kinase, p-Akt, p-PTP1B, p-PTEN, and P-ACL were evaluated as well.

\section{METHODS}

\section{Animal Treatments}

Animal procedures were approved by the Animal Care and Use Committee, faculty of Science, Anhui Medical University, in accordance with the International Guiding principles for Biomedical Research Involving animals of CIOMS.

An 8-wk-old C57BL/6J mice weighing between 11 and $14 \mathrm{~g}$, supplied by Beijing Vital River, whose foundation colonies were all introduced from Charles River Laboratories. The housing unit was maintained at constant temperature $\left(22-25^{\circ} \mathrm{C}\right)$ and humidity 50 $60 \%$, with a 12/12-h light/dark cycle (light on from 0600 to $1800 \mathrm{~h}$ ) with free access to tap water. After a week of adaptation, female mice were placed individually together with males at a ratio of $1: 2$. Mating was confirmed by the presence of a vaginal mucous plug the next morning, defined as gestational day zero (GD0). The total of successful mating was 41 over the same period. Among which 33 dams were administered with STZ (Sigma, Shanghai, China) to create GDM models, while the remaining 8 dams served as controls. The group of GDM mice was established by a single injection of streptozotocin as $40 \mathrm{mg} / \mathrm{kg} /$ per mouse on GD6, GD7, and GD8, respectively, for $3 \mathrm{~d}$ to ensure getting a successful GDM model (within $48 \mathrm{~h}$ glucose $>16.8$ $\mathrm{mmol} / \mathrm{l}$, defined as a successful model), and the control group mice received $0.9 \%$ saline (Sangon Biotech, Shanghai, China) alone. The successful models were 28 .

\section{Fetal Blood and Tissue Collection}

Fetal blood and tissue collection on GD18, half of GDM dams (14 litters of dams) and half of control dams (4 litters of dams) were randomly selected and the embryos were dissected by caesarean section after the pregnant mice were anaesthetized by an intraperitoneal injecting pentobarbital (Sangon Biotech, Shanghai, China) ( $150 \mathrm{mg} / \mathrm{kg}$ body weight). Specifically, three GDM fetal mice per litter and all control fetal mice were selected, which fetal weights, fetal glucose, crown-rump lengths, placental weights, and placental diameter were measured.

\section{Calorie Restriction}

Remaining survived dams were normally delivered. All of GDM and control offspring were fed ad libitum until $12 \mathrm{wk}$. At the $13^{\text {th }} \mathrm{wk}$, all offspring were further divided into three groups including two groups of GDM offspring and one group of control offspring: (i) control offspring with ad-libitum-fed (N-CON, $n=7$ ); (ii) GDM offspring fed ad- libitum (G-CON, $n=7$ ); (iii) GDM offspring treated with calorie restriction (G-CR, $n=7$ ); the proportion of male and female is halfto-half in each group. Each group of offspring contained seven mice (In order to spare, we took set up nine offspring per group in case), in which each GDM group contained nine offspring from the successful GDM models. Specifically, two mice per litter were selected based on the weight and glucose similarities, and randomly placed between two GDM groups. For the nine mice in the control group, the mice were randomly selected from the four litters of control offspring by 2-3 per litter. The intervention lasted $12 \mathrm{wk}$. The $\mathrm{N}-\mathrm{CON}$ and $\mathrm{G}-\mathrm{CON}$ groups were fed with standard chow that was accomplished by measuring the food intake by the mice every $24 \mathrm{~h}$. The food intake in CR group was reduced to $60 \%$ (comparing to the $\mathrm{N}-\mathrm{CON}$ group). The standard chow contains $20 \mathrm{kcal} \%$ protein, $70 \mathrm{kcal} \%$ carbohydrates, $10 \mathrm{kcal} \%$ fats, and a variety of essential vitamins and trace elements. Food intake of each mouse was recorded every day. Body weights were measured weekly. Fasting plasma glucose (FPG) levels were analyzed with a glucometer (Accu-Check Performa; Roche Diagnostics, Mannheim, Germany) every $2 \mathrm{wk}$ from the blood collected from the tail vein of the mice.

\section{Blood and Tissue Sample Collection}

On the final day of the experiment, blood samples were collected from the orbital venous plexus of 12 -h fasted and mice were anesthetized with ketamine $(100 \mathrm{mg} / \mathrm{kg})$, acepromazine $(10 \mathrm{mg} / \mathrm{kg})$, and xylazine $(100 \mathrm{mg} / \mathrm{kg})$ (Sangon Biotech, Shanghai, China). Whole blood was allowed to clot and then centrifuged at $3,000 \mathrm{~g}$ at $4^{\circ} \mathrm{C}$ for
20 min to obtain serum, which was kept at $-80^{\circ} \mathrm{C}$. Livers were immediately removed and rinsed with cold $0.9 \%$ saline. The liver was then chopped into pieces before being stored at $-80^{\circ} \mathrm{C}$.

\section{Biochemical Assays}

Serum levels of TCH, TG, HDL-C, and LDL-C were determined, respectively, using enzymatic kits (Nanjing Jiancheng Bioengineering Institute, Jiangsu, China).

\section{Enzyme-linked Immunosorbent Assay}

Insulin was analyzed using an enzyme-linked immunosorbent assay (ELISA) kit from CUSABIO Biotech Company (Wuhan, China) according to the manufacturer's protocol. Intra-assay Precision (Precision within an assay): $\mathrm{CV} \%<8 \%$, Inter-assay Precision (Precision between assays): CV \% $<10 \%$.

\section{Calculation of Insulin Resistance Index}

Insulin resistance was assessed using the homoeostasis model assessment of insulin resistance (HOMA-IR), was calculated using the following formula after treatment in experimental mice: (fasting insulin in $\mu \mathrm{IU} / \mathrm{ml} \times$ fasting glucose in $\mathrm{mmol} / \mathrm{l}) / 22.5$ (16).

\section{Analyses of Signaling Elements in Murine Livers}

Murine livers were homogenized in ice-cold lysis buffer. After $20 \mathrm{~min}$ splitting on ice the homogenates were centrifuged at $15,000 \mathrm{~g}$ for $15 \mathrm{~min}$ at $4^{\circ} \mathrm{C}$. Bicinchoninic acid (BCA) method (Thermo, Shanghai, China) was used for the determination of total proteins that were aliquoted, denatured and then frozen at $-80^{\circ} \mathrm{C}$ before Western blotting. Equal amounts of protein were subjected to sodium dodecyl sulfate polyacrylamide gel electrophoresis (SDS-PAGE) and transferred onto PVDF membranes. After incubation with appropriate primary and secondary antibodies, polyvinylidene fluoride (PVDF) membranes (Millipore, Shanghai, China) were washed by Tris-buffered saline-T (TBST) and targeted proteins were detected using enhanced chemifluorescence reagent. Obtained bands were quantified using Image Software, specifically, the membranes were incubated with antiserum against Akt (Abcam, Shanghai, China), PI3K (Abcam), PTP1B (Abcam), PTEN (Cell Signaling Technology, Shanghai, China), ACL (Cell Signaling Technology), phosphor-Akt (Cell Signaling Technology), phosphor-PTEN (Cell Signaling Technology), phosphor-PTP1B (Cell Signaling Technology), and phosphor-ACL (Abcam), respectively. Each sample was analyzed an average of three separate times involving different gels.

\section{Statistical Analysis}

Data were presented as mean \pm SEM for the baseline data of mice. Statistical analysis was performed using unpaired student's $t$-test for fetal body weights, fetal glucose levels, crown-rump lengths, and placental weights, placental diameters, and weights, glucose levels before intervention. The sex ratios were analyzed by Chi-square test. The effects of food intakes, body weights, and plasma glucose levels after intervention were analyzed by repeated measures two-way ANOVA followed by Tukey's multiple comparisons test. All other measured parameters were compared using regular two-way ANOVA.

All statistical analyses were performed using SPSS 16.0. A value of $P$ $<0.05$ was considered to be statistically significant. Graphics were constructed using GraphPad Prism 6 (GraphPad Software, San Diego, CA)

\section{RESULTS}

Weight, Glucose, and General Growth of Fetal between GDM and Control Groups

The growth of fetal mice was generally normal in control group. We found GDM dams give birth of 5-7 pups by one litter (sex ratio: male/female $=1.05$ ), however control dams give birth of 8-9 pups per litter (sex ratio was male : female $=1.17$ ), there were no significant differences in genders. Compared with the control group, GDM fetuses obtained significant decreases in the body weight, crown-rump length and placental weight, in addition, GDM fetuses had a higher glucose levels than those in control group $(P<0.05)$ (Table 1$)$. 
Table 1. Weight, glucose, and general growth between the fetal of GDM and control groups, the weight and glucose before intervention ( $x \pm$ SEM)

\begin{tabular}{lcccccccc}
\hline & n/litters & $\begin{array}{c}\text { Fetal body } \\
\text { weight }(\mathrm{g})\end{array}$ & $\begin{array}{c}\text { Fetal glucose } \\
(\mathrm{mmol} / \mathrm{l})\end{array}$ & $\begin{array}{c}\text { Crown-rump } \\
\text { length }(\mathrm{mm})\end{array}$ & $\begin{array}{c}\text { Placental } \\
\text { weight }(\mathrm{mg})\end{array}$ & $\begin{array}{c}\text { Placental } \\
\text { diameter (mm) }\end{array}$ & $\begin{array}{c}\text { Weight (before } \\
\text { intervention) }\end{array}$ & $\begin{array}{c}\text { Glucose (before } \\
\text { intervention) }\end{array}$ \\
\hline Control offspring & $33 / 4$ & $1.05 \pm 0.01$ & $2.30 \pm 0.08$ & $21.58 \pm 0.14$ & $68.7 \pm 1.41$ & $7.11 \pm 0.15$ & $23.4 \pm 1.32$ & $5.78 \pm 0.08$ \\
GDM offspring & $42 / 14$ & $0.80 \pm 0.02^{*}$ & $4.65 \pm 0.14^{*}$ & $18.65 \pm 0.22^{*}$ & $59.9 \pm 2.43^{*}$ & $6.79 \pm 0.12$ & $24.4 \pm 0.94$ & $7.22 \pm 0.34^{*}$ \\
\hline
\end{tabular}

GDM, gestational diabetes mellitus. Compared with control group, ${ }^{*} P<0.05$

\section{Murine Body Weights}

No significant differences in average body weights were observed between GDM offspring and control offspring before intervention (Table 1). During weeks of 3-12, the body weights of G-CR group (GDM offspring treated with calorie restriction) were significantly lower than those in G-CON (GDM offspring fed ad-libitum) $(P<0.05)$. During weeks of $4-12$, the body weights of N-CON group (control offspring fed ad-libitum) remained stable and higher than those of G-CR mice $(P<0.05)$ (Figure 1a). The final average body weights of G-CR mice were lower than those in other groups (Figure 1a).

\section{Plasma Glucose}

GDM offspring had higher levels of average plasma glucose than those in control offspring before intervention $(P<$ 0.05) (Table 1). After $4 \mathrm{wk}$ of intervention, there were no significant differences in glucose levels between G-CR and $\mathrm{N}$-CON groups. But the glucose levels of G-CON mice had a greater fluctuation, by the profile analysis using Tukey method, we found that the blood glucose levels decreased slightly at week 2, and came back to the levels before the intervention, and that blood glucose levels were higher at week 10 to week 12 than those in G-CON at the same time and keep stable. While plasma glucose levels had a statistically significant difference at week 10-12 $(P<0.05)$; More importantly, the G-CR group tend to become stabilized (Figure 1b). After $12 \mathrm{wk}$ of intervention, the final average glucose levels in G-CR mice were lower than those of G-CON mice $(P<0.05)$, and there was no difference compared with $\mathrm{N}-\mathrm{CON}$ mice.

\section{Food Intake}

There were no significant differences in everyday food intakes between G-CON group and N-CON group, however, the everyday food intakes in G-CR group were lower than those in G-CON group $(P<0.05)$ (Figure $1 \mathrm{c})$.

\section{Serum Lipid Profile after 12 wk of Intervention}

The serum levels of TCH, TG, HDL-C, and LDL-C were demonstrated in Figure 2. Compared with $\mathrm{N}-\mathrm{CON}$ mice, G-CON mice had significant increase in $\mathrm{TCH}$ (23\%), TG (29\%), and LDL-C (57\%), but a notable decrease in HDL-C (44\%). Compared with G-CON, CR elicited a significant decrease in TCH (72\%), TG (34\%), and LDL-C (63\%), respectively, but a significant increase in HDL-C (45\%) implicating that $\mathrm{CR}$ can reverse the adverse lipid metabolism in GDM offspring. a

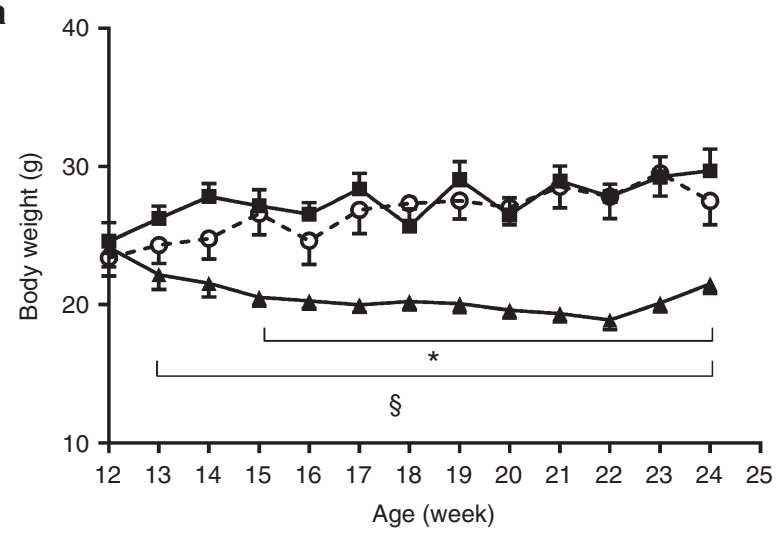

b

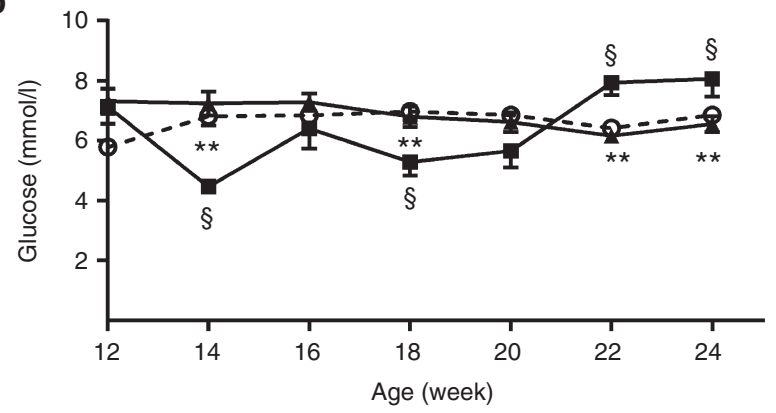

C

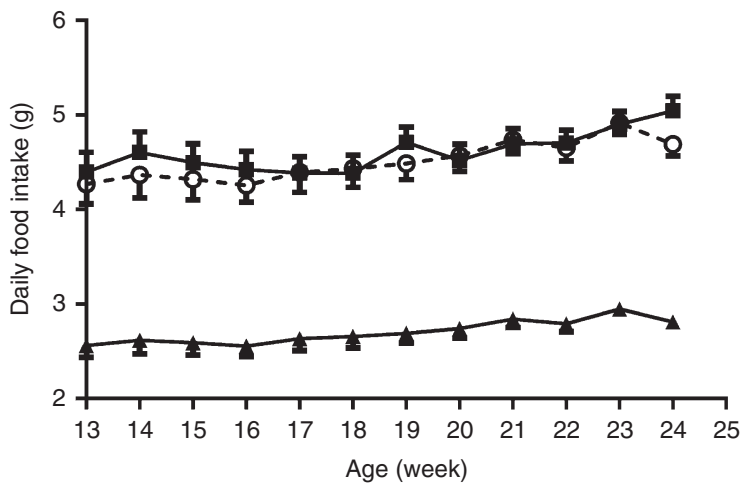

Figure 1. Body weights (a), glucose levels (b) and daily food intake (c) of control offspring and GDM offspring with ad-libitum-fed or calorie restriction(CR). Assays of body weights, glucose levels and daily food intake were performed at least three times per experiment, and the average values are presented. Values are mean \pm SE for 7 animals/group. ${ }^{*} P<0.05 \mathrm{~N}-\mathrm{CON}$ vs. G-CR; $\S P<0.05 \mathrm{G}-\mathrm{CON}$ vs. G-CR; ${ }^{* *} P<0.05 \mathrm{~N}-\mathrm{CON}$ vs.

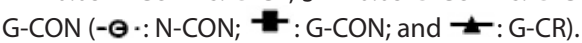

Serum Insulin Levels and Insulin Resistance Index after 12 wk of Intervention

Compared with $\mathrm{N}-\mathrm{CON}$ mice, the G-CON mice had a significantly higher levels of insulin $(P<0.05)$, in which was 


\section{Articles | Liet al.}

about a $248 \%$ increase. Furthermore, after $12 \mathrm{wk}$ of intervention, the insulin levels in G-CR mice were lower than those in G-CON mice $(P<0.05)$ (Figure 3a). Additionally, HOMA-IR in the offspring of G-CON group was significantly higher than those in N-CON and G-CR groups respectively, at 24 wk of age $(P<0.05)$. However, there were no significant differences between G-CR group and C-CON group (Figure $3 \mathbf{b}$ ).

\section{Effect of Caloric Restriction on Insulin-signaling in GDM Livers}

In this study, we found that CR enhances Akt activity. Figure 4a showed that total Akt levels were 27.7\% lower in livers from G-CR mice compared with livers from N-CON group. However, phosphorylations of Akt were increased by $21.7 \%$ in G-CR mice compared with those in G-CON group, and the levels of p-Akts in G-CR mice were higher than those in N-CON mice (Figure 4b). Notably, we found phosphorylations of Akts in G-CR mice (p-Akt normalized by total Akt) were increased by $62.7 \%$ compared with those in $\mathrm{N}-\mathrm{CON}$ mice. Meanwhile, GDM offspring (G-CON) had a lower levels of phosphorylations of Akts (p-Akt normalized by total Akt) compared with N-CON mice (Figure 4c).

Since PI3K is a major upstream regulator of Akt, we detected the levels of PI3K and p-PI3K, respectively. But we found nonsignificant differences among the three murine groups regarding PI3K and p-PI3K (Figure 4d-f).

Additionally, we found that total PTEN protein levels in G-CON mice were much higher than those in G-CR group, or mildly higher than those in N-CON group. Similarly, PTEN phosphorylation was increased $\sim 2.36$-fold in G-CON compared with that in $\mathrm{N}-\mathrm{CON}$ mice, however, after intervention, the p-PTEN levels of G-CR group reduced to the levels in a

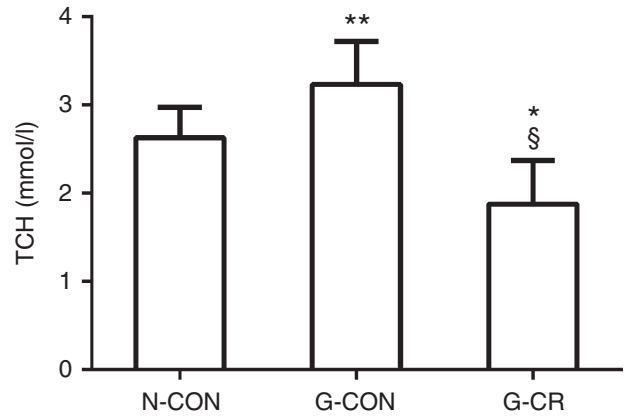

C

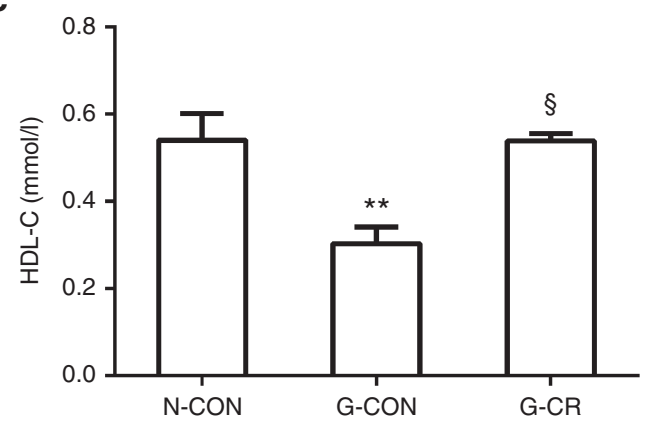

b

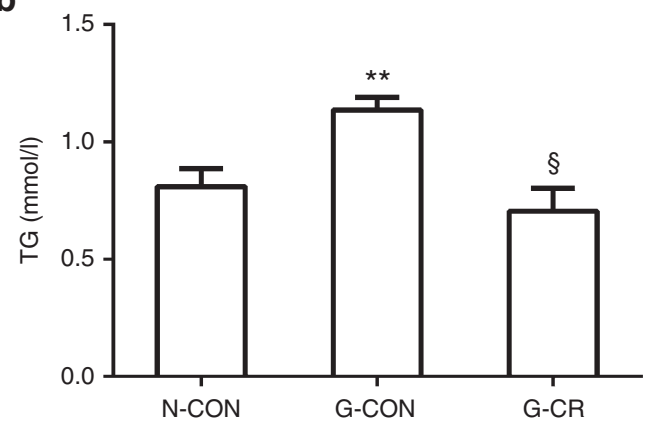

d

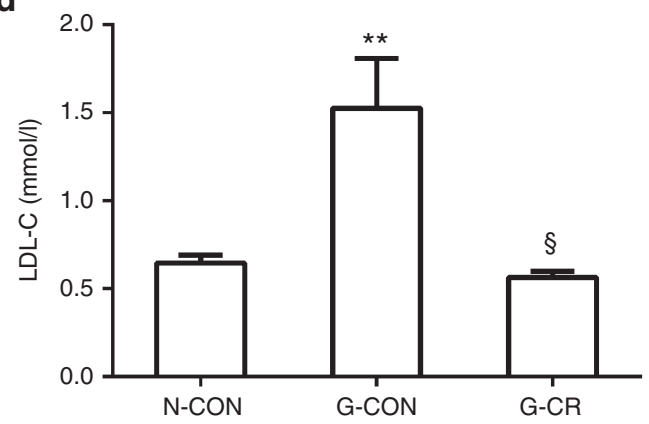

Figure 2. TCH levels (a); TG levels(b); HDL-C levels(c) and LDL-C levels(d) in control murine offspring and gestational diabetes mellitus (GDM) murine offspring with ad libitum-fed or calorie restriction (CR).Values are mean \pm SEM for 7 animals/group.* $P<0.05 \mathrm{~N}-\mathrm{CON}$ vs. G-CR; $\S P<0.05 \mathrm{G}-\mathrm{CON}$ vs. G-CR; ${ }^{*} \mathrm{P}<0.05 \mathrm{~N}-\mathrm{CON}$ vs. G-CON.
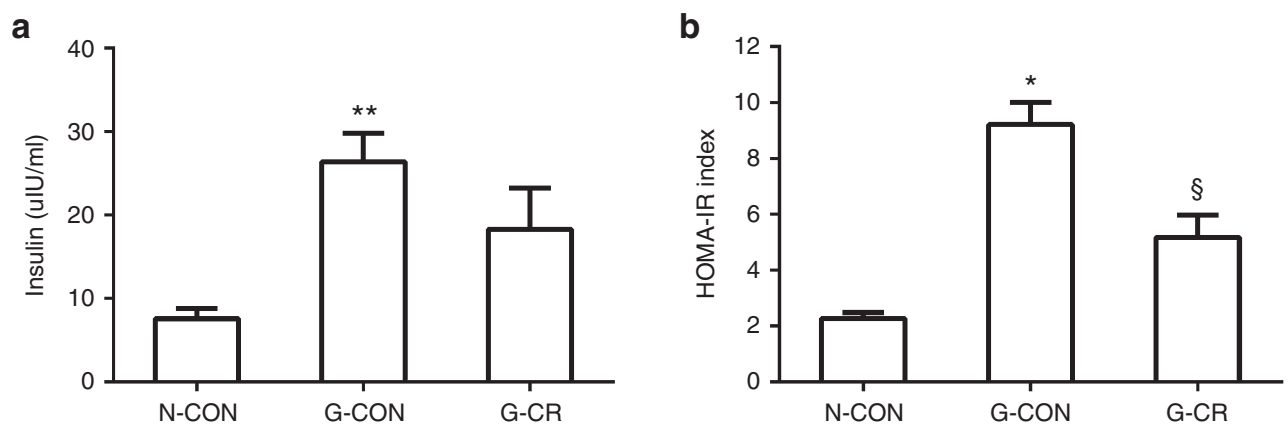

Figure 3. Insulin levels (a) and insulin resistance index (b) in control murine offspring and gestational diabetes mellitus (GDM) murine offspring with ad libitum-fed or calorie restriction (CR). Values are mean \pm SEM for 7 animals/group.*P< $0.05 \mathrm{~N}-\mathrm{CON}$ vs. G-CR; $\S P<0.05 \mathrm{G}-\mathrm{CON}$ vs. G-CR; *** $<0.05 \mathrm{~N}-\mathrm{CON}$ vs. G-CON. 
a

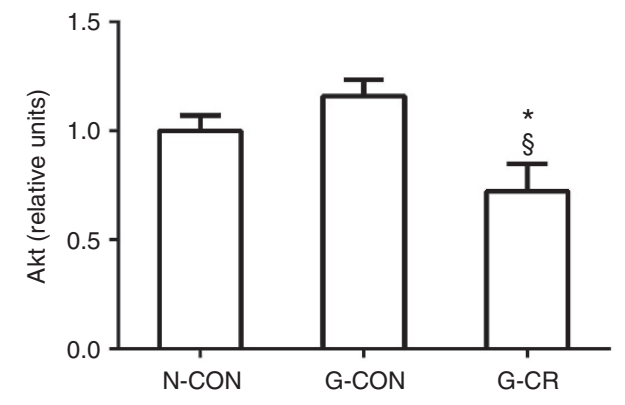

b

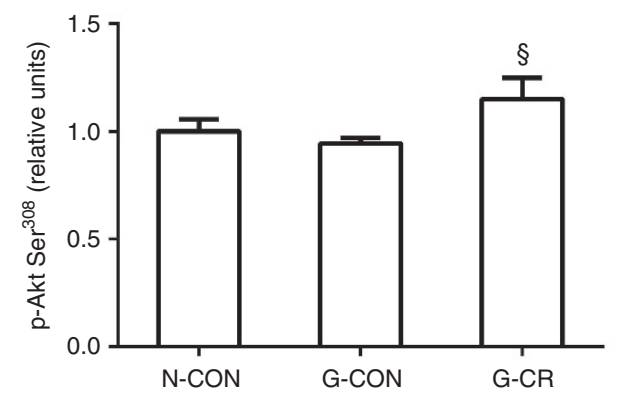

d

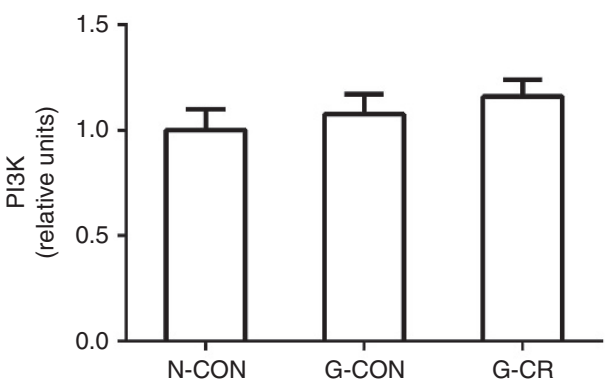

e

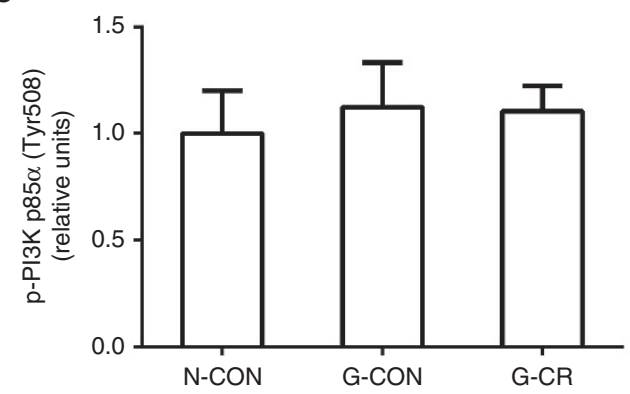

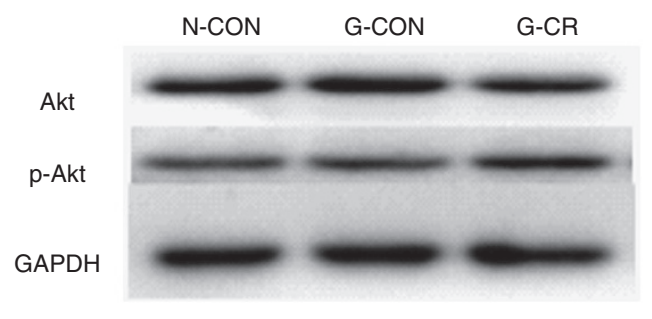

c
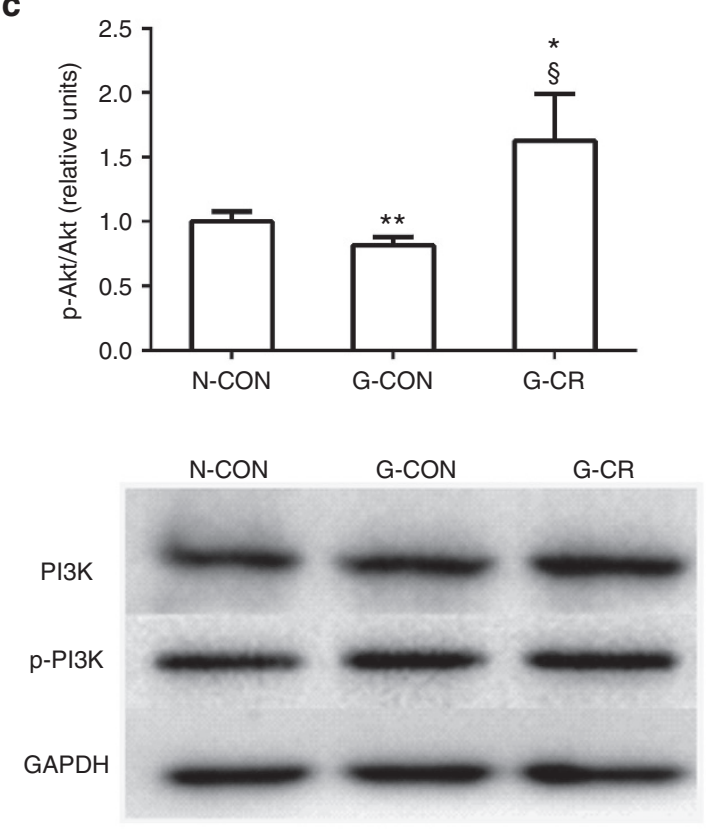

f

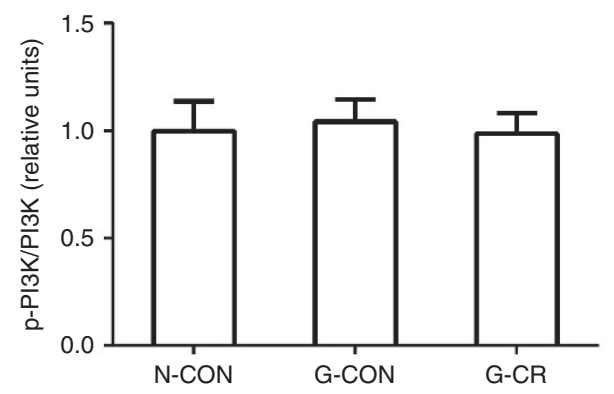

Figure 4. $A k t(\mathbf{a}), \mathrm{p}-A k t \operatorname{ser}^{308}(\mathbf{b}), \mathrm{p}-\mathrm{Akt}$ normalized by total $\mathrm{Akt}(\mathbf{c}), \mathrm{PI} 3 \mathrm{~K}(\mathbf{d}), \mathrm{p}-\mathrm{PI} 3 \mathrm{~K}$ Tyr $\mathrm{r}^{508}(\mathbf{e})$ and $\mathrm{p}-\mathrm{PI} 3 \mathrm{~K}$ normalized by total PI3K(f) protein levels in control murine offspring and gestational diabetes mellitus (GDM) murine offspring with ad libitum-fed or calorie restriction (CR). The signaling proteins were determined by immunoblot analysis and normalized to glyceraldehyde 3-phosphate dehydrogenase (GAPDH). Data are presented as fold change over the $\mathrm{N}-\mathrm{CON}$ group. Values are mean $\pm \mathrm{SEM}$ for 7 animals/group.*P< $0.05 \mathrm{~N}-\mathrm{CON}$ vs. G-CR; $\S P<0.05 \mathrm{G}-\mathrm{CON}$ vs. G-CR; ${ }^{* *} P<0.05 \mathrm{~N}-\mathrm{CON}$ vs. G-CON. PI3K, phosphotidylinositol-3-kinase; PTEN, phosphatase and tensin homologue deleted on chromosome 10; PTTP1B, protein tyrosine phosphatase 1B.

N-CON group, which decreased twofold compared with those in G-CON group Figure 5a-c .

Figure 5d revealed that PTP1B protein levels were reduced in G-CR mice compared with those in G-CON mice. However, p-PTP1B levels did not differ among three groups (Figure 5e).

Figure 6a showed that total ACL was 54.7\% lower in G-CON mice compared with those in $\mathrm{N}-\mathrm{CON}$ mice. More importantly, we found that CR led to a significant $(40.2 \%)$ reduction in the phosphorylation levels of ACL comparing to those in N-CON mice $(P<0.05)$. Similarly, ACL phosphorylation levels were decreased $37.9 \%$ in G-CR mice comparing to those in G-CON group $(P<0.05)$ (Figure $6 \mathbf{b}$ ).

\section{DISCUSSION}

GDM is associated with adiposity, insulin resistance, and a great risk for developing obesity and diabetes in the offspring. 


\section{Articles $\mid$ Lietal.}

a

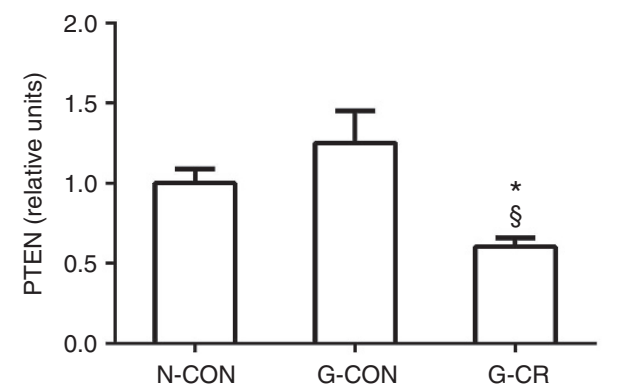

b

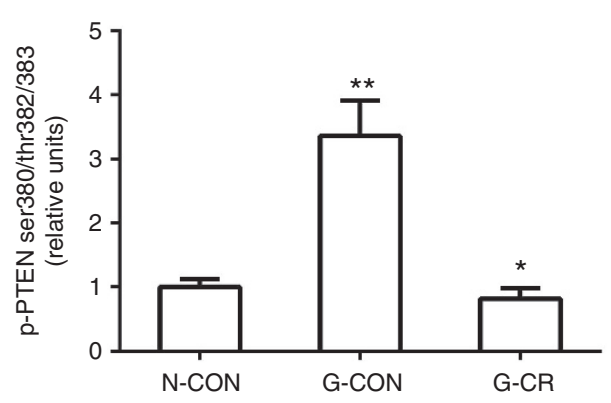

d

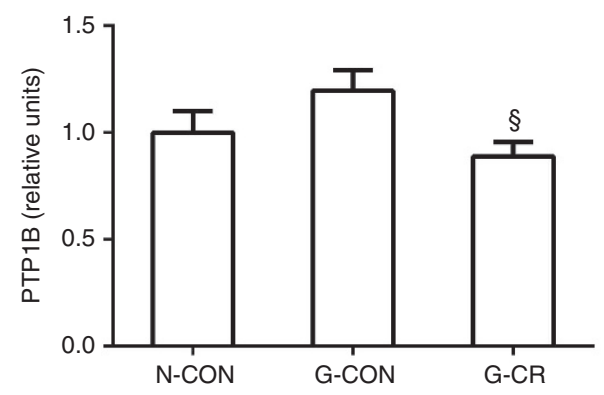

e

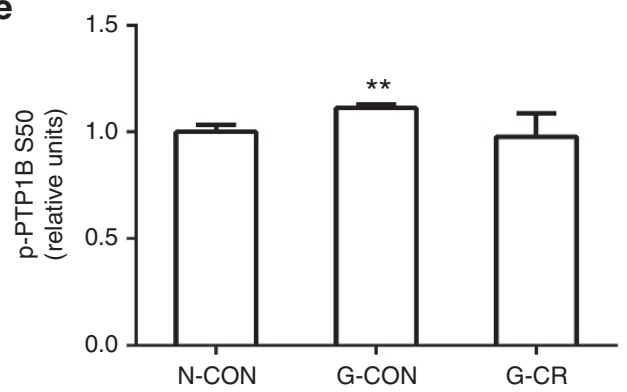

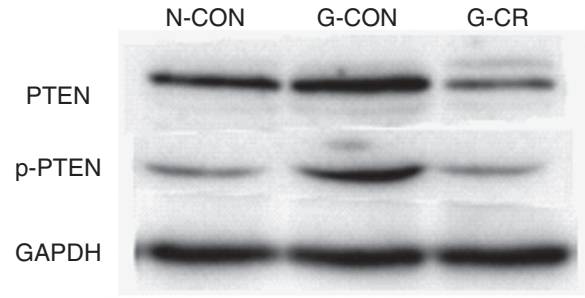

C
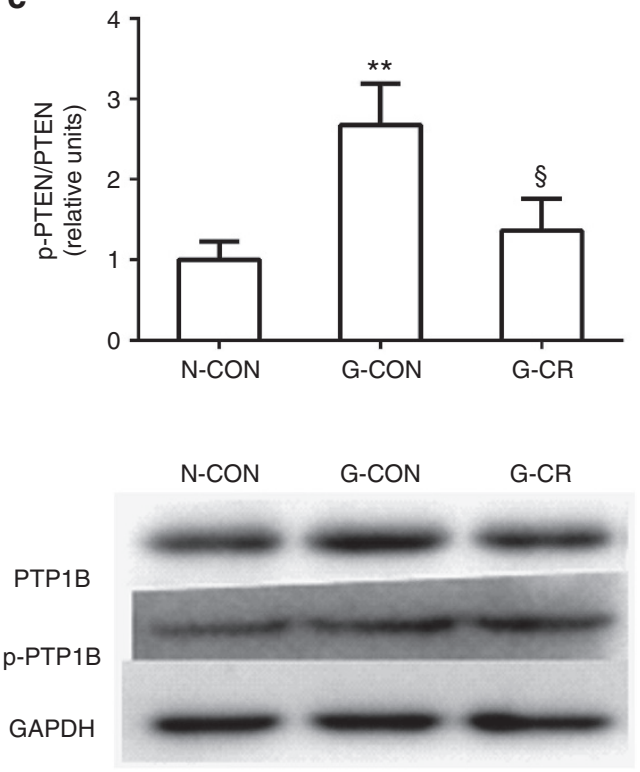

$\mathbf{f}$

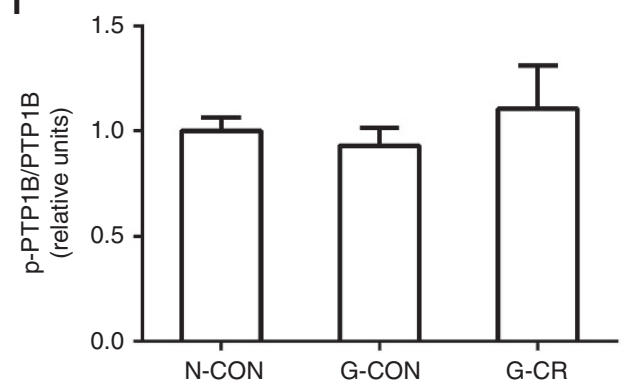

Figure 5. PTEN (a), p-PTEN ser ${ }^{380} /$ thr $^{382 / 383}$ (b), p-PTEN normalized by total PTEN (c), PTP1B(d), p-PTP1B S ${ }^{50}$ (e) and p-PTP1B normalized by total PTP1B (f) protein levels in control murine offspring and gestational diabetes mellitus (GDM) murine offspring with ad libitum-fed or calorie restriction (CR). The signaling proteins were determined by immunoblot analysis and were normalized to glyceraldehyde 3-phosphate dehydrogenase (GAPDH). Data are presented as fold change over the N-CON group. Values are mean \pm SE for 7 animals/group. ${ }^{*} P<0.05 \mathrm{~N}-\mathrm{CON}$ vs. G-CR; $\S P<0.05 \mathrm{G}-\mathrm{CON}$ vs. G-CR; ${ }^{* *} P<0.05$ $\mathrm{N}-\mathrm{CON}$ vs. G-CON. PTEN, phosphatase and tensin homologue deleted on chromosome 10; PTTP1B, protein tyrosine phosphatase 1B.

It is known that CR can not only extend life span $(17,18)$ but also protect against cardiovascular diseases $(19,20)$, which includes increasing insulin sensitivity $(7,21)$, prevention of cardiomyocyte apoptosis (22). Our results showed that GDM resulted in significant decrease in the fetal body weights, which is consistent with Dabelea's finding that indicated that exposure to this diabetic intrauterine environment also causes changes in fetal growth, therefore predispose to abnormal glucose tolerance and insulin resistance in adulthood, even in the absence of macrosomia at birth (23). Moreover, we found that in the medium and long effects, the weights of GDM offspring could became normal, suggesting that although the existence of intrauterine and postnatal growth retardations that may cause postnatal catch-up growth, the catch-up growth may cause long-term adverse effects on the body, leading to changes in development and in insulin sensitivity $(24,25)$. In addition, we found GDM murine offspring had high levels of insulin and high insulin resistance index, resulting in a greater fluctuation of glucose and an abnormal lipid metabolism than those in control murine offspring. While based on this study, the 
a
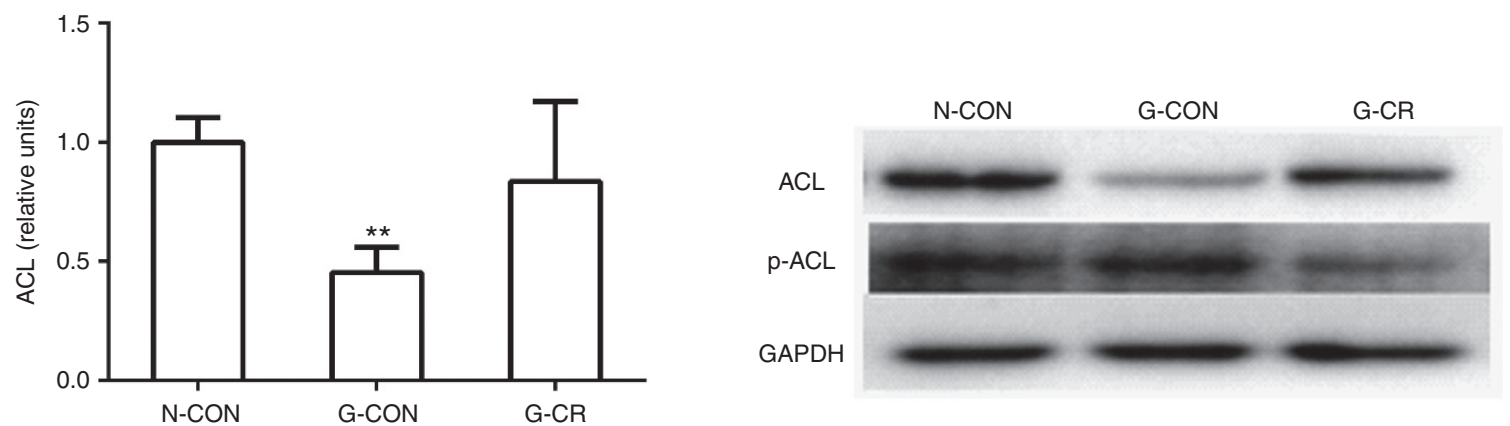

b
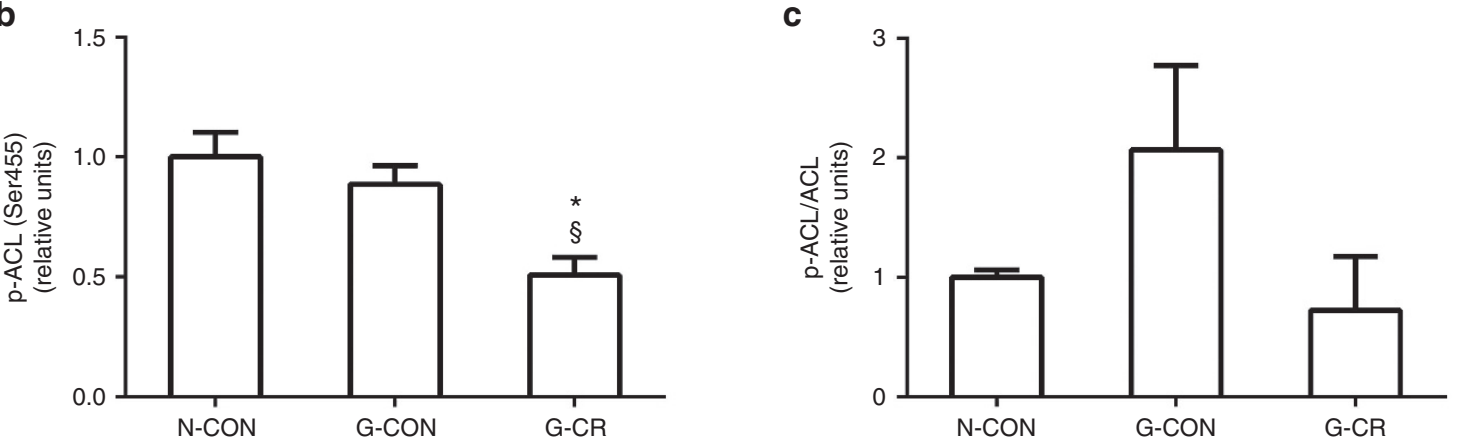

Figure 6. $\mathrm{ACL}(\mathbf{a}), \mathrm{p}-\mathrm{ACL} \operatorname{ser}^{454}(\mathbf{b})$ and $\mathrm{p}-\mathrm{ACL}$ normalized by total $\mathrm{ACL}$ (c) protein levels in control murine offspring and gestational diabetes mellitus (GDM) murine offspring with ad libitum-fed or calorie restriction (CR). The signaling proteins were determined by immunoblot analysis and were normalized to glyceraldehyde 3-phosphate dehydrogenase (GAPDH). Data are presented as fold change over the N-CON group. Values are mean \pm SE for 7 animals/group. ${ }^{*} P<0.05 \mathrm{~N}-\mathrm{CON}$ vs. G-CR; $§ P<0.05 \mathrm{G}-\mathrm{CON}$ vs. G-CR; ${ }^{* *} P<0.05 \mathrm{~N}-\mathrm{CON}$ vs. G-CON.

glucose levels of GDM offspring could became stable through caloric restriction, and CR could ameliorate the abnormal lipid metabolism as well. Moreover, we found that GDM offspring probably have formed insulin resistance, while caloric restriction was associated with improved insulin sensitivity. These results implied that limiting energy intake may be necessary for reducing insulin resistance in GDM offspring.

In liver, CR could reduce lipogenesis (26), a rate-limiting step in hepatic fat metabolism. This reprogram decreases the lipid biosynthesis including alterations of phosphorylations of ACL after insulin stimulation (27). However, the specific cellular mechanism(s) leading to the reduction of lipogenesis in GDM offspring was uncertain.

The PI3K/ PKB/Akt signaling pathway play a pivotal role in insulin sensitivity. Among which PI3K activation was essential for fat metabolism $(28,29)$ and might be a possible mediator for the effects of CR on insulin sensitivity. But in this report, we did not find a significant change in PI3K activity/phosphorylation. In addition, Davidson et al. did not find a significant change either in IRS- 1 activity, or in IRS- 2 and PI3K activities by CR (30), which is consistent with our findings. Considering the lack of a significant effect of CR on PI3K and its upstream regulators, we have become interested in downstream targets of PI3K in insulin signaling.

In this study, we found a significant decrease in total Akt protein levels from GDM offspring with CR, which is similar to the report from McCurdy and his colleagues report that there was a reduction in total Akt1 protein expression with no effect on total Akt2 after brief CR (31). GDM offspring had a lower phosphorylation of Akt, which was greatly elevated by CR compared with ad-libitum-fed groups. Thus, these findings led us hypothesize that CR would enhance insulin signaling by increasing activation of Akt. Other articles also suggested that Akt-deficient mice may change insulin signaling (32). Especially the mice deficient in Akt2 are impaired in the ability of insulin to lower blood glucose because of defects in the action of the hormone on liver (33). Besides, 3-phosphoinositide-dependent kinase 1 (PDK1) was a primary activator of Akt at the plasma membrane, which is tightly related to activation of membrane-associated Akt at threonine 308 in the activation-loop of the kinase domain and Ser473 in the carboxy-terminal regulatory region (34-36). The primary function of PTEN was exerted on the dephosphorylation of phosphatidylinositol 3,4,5-trisphosphate (PIP3) that activates PDK-1, and subsequently influence in the activation of Akt in the insulin signaling pathway (37). Similar to the PTEN, PTP1B, as a negative regulator, inhibited the Akt activation through a pathway along a PI3K/PDK1/Akt axis (38). In our data, GDM offspring had higher protein levels of total PTEN, p-PTEN, and p-PTP1B comparing to those in N-CON group, while CR could decrease the levels of total PTEN, p-PTEN, and did not change the levels of p-PTP1B. We can conclude that CR may ameliorate the insulin signaling among GDM offspring by decreasing the activation of PTEN.

ACL was a cytosolic enzyme most highly expressed in lipogenic tissues such as liver and white adipose tissue (39). ACL plays a fundamental role in lipogenesis and steroidogenesis. Its activation provides the building blocks for fatty acid 


\section{Articles | Lietal.}

biosynthesis and cholesterol (40). In this study, we found that CR could decrease the activation of ACL among GDM offspring. The possible explanation was that the phosphorylation of Akt may change the downstream ACL activation, and therefore, inhibited the lipogenesis and steroidogenesis. We speculated that the favorable effect of CR on insulin action in liver was associated with the suppression of hepatic fat accumulation and free fatty acid level in plasma.

In summary, the present investigation suggested that GDM exerts a strong effect on the subsequent development of obesity and hepatic insulin resistance in GDM offspring. The hepatic insulin resistance was associated with a specific abnormality in PTEN phosphorylation. The defect in Akt activation was also associated with greater TG, TC, and insulin levels. However, $\mathrm{CR}$ may prevent the development of hepatic insulin resistance in GDM offspring by improving the phosphorylation of Akt and lowering the activation of PTEN. Therefore, CR may be a useful therapy for ameliorating or preventing the adverse outcomes such as insulin resistance in GDM offspring.

\section{STATEMENT OF FINANCIAL SUPPORT}

This work is supported by grants from the National Natural Science Foundation of China (No. 81373011 by C-L.H. and No. 81570786 by K.C.).

Disclosure: There are no disclosures.

\section{REFERENCES}

1. Reece EA, Leguizamón G, Wiznitzer A. Gestational diabetes: the need for a common ground. Lancet 2009;373:1789-97.

2. Ajala O, Jensen LA, Ryan E, Chik C. Women with a history of gestational diabetes on long-term follow up have normal vascular function despite more dysglycemia, dyslipidemia, and adiposity. Diabetes Res Clin Pract 2015;110:309-14.

3. Sreelakshmi PR, Nair S, Soman B, Alex R, Vijayakumar K, Kutty VR. Maternal and neonatal outcomes of gestational diabetes: a retrospective cohort study from Southern India. J Family Med Prim Care 2015;4:395-8.

4. Pereira TJ, Moyce BL, Kereliuk SM, Dolinsky VW. Influence of maternal overnutrition and gestational diabetes on the programming of metabolic health outcomes in the offspring: experimental evidence. Biochem Cell Biol 2014: $1-14$.

5. Quilter CR, Cooper WN, Cliffe KM, et al. Impact on offspring methylation patterns of maternal gestational diabetes mellitus and intrauterine growth restraint suggest common genes and pathways linked to subsequent type 2 diabetes risk. FASEB J 2014;28:4868-79.

6. Pettitt DJ, Nelson RG, Saad MF, Bennett PH, Knowler WC. Diabetes and obesity in the offspring of Pima Indian women with diabetes during pregnancy. Diabetes Care 1993;16:310-4.

7. Meehan CA, Cochran E, Mattingly M, Gorden P, Brown RJ. Mild caloric restriction decreases insulin requirements in patients with type 2 diabetes and severe insulin resistance. Medicine (Baltimore) 2015;94:e1160.

8. Lara-Castro C, Newcomer BR, Rowell J, et al. Effects of short-term very low-calorie diet on intramyocellular lipid and insulin sensitivity in nondiabetic and type 2 diabetic subjects. Metabolism 2008;57:1-8.

9. Speakman JR, Mitchell SE. Caloric restriction. Mol Aspects Med 2011;32:159-221.

10. Ravussin E, Redman LM, Rochon J, et al.; CALERIE Study Group. A 2-year randomized controlled trial of human caloric restriction: feasibility and effects on predictors of health span and longevity. J Gerontol A Biol Sci Med Sci 2015;70:1097-104.

11. Dolinsky VW, Dyck JR. Calorie restriction and resveratrol in cardiovascular health and disease. Biochim Biophys Acta 2011;1812:1477-89.

12. Wang P, Zhang RY, Song J, et al. Loss of AMP-activated protein kinase- $\alpha 2$ impairs the insulin-sensitizing effect of calorie restriction in skeletal muscle. Diabetes 2012;61:1051-61.
13. Bales CW, Kraus WE. Caloric restriction: implications for human cardiometabolic health. J Cardiopulm Rehabil Prev 2013;33:201-8.

14. Schenk S, McCurdy CE, Philp A, et al. Sirtl enhances skeletal muscle insulin sensitivity in mice during caloric restriction. J Clin Invest 2011;121:4281-8.

15. García-San Frutos M, Fernández-Agulló T, Carrascosa JM, et al. Involvement of protein tyrosine phosphatases and inflammation in hypothalamic insulin resistance associated with ageing: effect of caloric restriction. Mech Ageing Dev 2012;133:489-97.

16. Matthews DR, Hosker JP, Rudenski AS, Naylor BA, Treacher DF, Turner RC. Homeostasis model assessment: insulin resistance and betacell function from fasting plasma glucose and insulin concentrations in man. Diabetologia 1985;28:412-9.

17. Molin M, Demir AB. Linking peroxiredoxin and vacuolar-ATPase functions in calorie restriction-mediated life span extension. Int J Cell Biol 2014;2014:913071.

18. Rona G, Herdeiro R, Mathias CJ, Torres FA, Pereira MD, Eleutherio E. CTT1 overexpression increases life span of calorie-restricted Saccharomyces cerevisiae deficient in Sod1. Biogerontology 2015;16:343-51.

19. Kroeger CM, Klempel MC, Bhutani S, Trepanowski JF, Tangney CC, Varady KA. Improvement in coronary heart disease risk factors during an intermittent fasting/calorie restriction regimen: relationship to adipokine modulations. Nutr Metab (Lond) 2012;9:98.

20. Weiss EP, Fontana L. Caloric restriction: powerful protection for the aging heart and vasculature. Am J Physiol Heart Circ Physiol 2011;301:H1205-19.

21. Kirk E, Reeds DN, Finck BN, et al. Dietary fat and carbohydrates differentially alter insulin sensitivity during caloric restriction. Gastroenterology 2009;136:1552-60.

22. Lee CK, Allison DB, Brand J, Weindruch R, Prolla TA. Transcriptional profiles associated with aging and middle age-onset caloric restriction in mouse hearts. Proc Natl Acad Sci USA 2002;99:14988-93.

23. Dabelea D. The predisposition to obesity and diabetes in offspring of diabetic mothers. Diabetes Care 2007;30 Suppl 2:S169-74.

24. Deng HZ, Deng H, Cen CQ, Chen KY, Du ML. Post-receptor crosstalk between growth hormone and insulin signal in rats born small for gestational age with catch-up growth. PLoS One 2014;9:e100459.

25. Alves JG, Vasconcelos SA, de Almeida TS, Lages R, Just E. Influence of catch-up growth on abdominal fat distribution in very low birth weight children-cohort study. J Pediatr Endocrinol Metab 2015; 28:153-6.

26. Kuhla A, Hahn S, Butschkau A, Lange S, Wree A, Vollmar B. Lifelong caloric restriction reprograms hepatic fat metabolism in mice. J Gerontol A Biol Sci Med Sci 2014;69:915-22.

27. Wu X, Chen $\mathrm{K}$, Williams KJ. The role of pathway-selective insulin resistance and responsiveness in diabetic dyslipoproteinemia. Curr Opin Lipidol 2012;23:334-44.

28. Han $\mathrm{C}$, Wei $\mathrm{S}, \mathrm{He} \mathrm{F}$, et al. The regulation of lipid deposition by insulin in goose liver cells is mediated by the PI3K-AKT-mTOR signaling pathway. PLoS One 2015;10:e0098759.

29. Staff PG. Correction: Maf1 is a novel target of PTEN and PI3K signaling that negatively regulates oncogenesis and lipid metabolism. PLOS Genetics Staff 2015;11:e1005055.

30. Davidson RT, Arias EB, Cartee GD. Calorie restriction increases muscle insulin action but not IRS-1-, IRS-2-, or phosphotyrosine-PI 3-kinase. Am J Physiol Endocrinol Metab 2002;282:E270-6.

31. McCurdy CE, Davidson RT, Cartee GD. Brief calorie restriction increases Akt2 phosphorylation in insulin-stimulated rat skeletal muscle. Am J Physiol Endocrinol Metab 2003;285:E693-700.

32. Reynolds THt, Merrell E, Cinquino N, Gaugler M, Ng L. Disassociation of insulin action and Akt/FOXO signaling in skeletal muscle of older Akt-deficient mice. Am J Physiol Regul Integr Comp Physiol 2012;303:R1186-94.

33. Cho H, Mu J, Kim JK, et al. Insulin resistance and a diabetes mellitus-like syndrome in mice lacking the protein kinase Akt2 (PKB beta). Science 2001;292:1728-31.

34. Park H, Lee S, Shrestha P, et al. AMIGO2, a novel membrane anchor of PDK1, controls cell survival and angiogenesis via Akt activation. J Cell Biol 2015;211:619-37. 
35. Pearn L, Fisher J, Burnett AK, Darley RL. The role of PKC and PDK1 in monocyte lineage specification by Ras. Blood 2007;109:4461-9.

36. Yang J, Cron P, Thompson V, et al. Molecular mechanism for the regulation of protein kinase B/Akt by hydrophobic motif phosphorylation. Mol Cell 2002;9:1227-40.

37. Maehama T, Dixon JE. The tumor suppressor, PTEN/MMAC1, dephosphorylates the lipid second messenger, phosphatidylinositol 3,4,5-trisphosphate. J Biol Chem 1998;273:13375-8.
38. Shibata E, Kanno T, Tsuchiya A, et al. Free fatty acids inhibit protein tyrosine phosphatase 1B and activate Akt. Cell Physiol Biochem 2013;32: 871-9.

39. Wang Q, Li S, Jiang L, et al. Deficiency in hepatic ATP-citrate lyase affects VLDL-triglyceride mobilization and liver fatty acid composition in mice. J Lipid Res 2010;51:2516-26.

40. Lemus HN, Mendivil CO. Adenosine triphosphate citrate lyase: emerging target in the treatment of dyslipidemia. J Clin Lipidol 2015;9:384-9. 